\title{
Preparation of Translationally Cold Neutral Molecules
}

\author{
Giulia Di Domenicantonio, Benjamin Bertsche, and Andreas Osterwalder
}

\begin{abstract}
Efforts at EPFL to obtain translationally cold neutral molecules are described. Active deceleration of polar molecules is performed by confining the molecules in moving three-dimensional electrostatic traps, and by appropriately choosing the velocity of those traps. Alternatively, cold molecules can be obtained by velocity filtering. Here, the velocity of the molecules is not changed, but instead the cold molecules are extracted from a thermal sample by using the competition between the electrostatic force and the centrifugal force inside a bent electrostatic guide for polar molecules.
\end{abstract}

Keywords: Cold molecules $\cdot$ Polar molecules $\cdot$ Stark deceleration $\cdot$ Velocity filtering

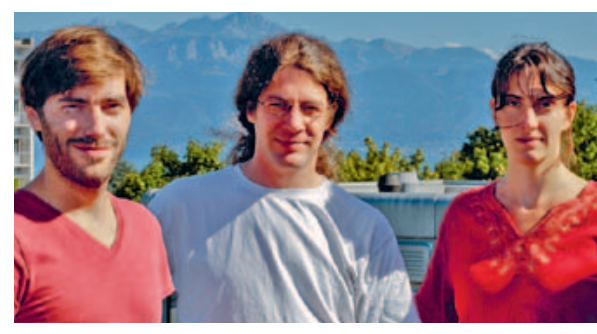

B. Bertsche, A. Osterwalder,

G. Di Domenicantonio

\section{Introduction}

The preparation, isolation, and control of translationally cold neutral molecules in the gas phase is an experimentally complex task that has become possible only during the past two decades. ${ }^{[1]}$ Among the main developments that triggered interest in cold molecules was the success of cold atoms research that truly revolutionized the field of atomic physics. One may hope that porting the concepts and methods from cold and ultracold atomic physics, with temperatures well below $1 \mathrm{~K}$, into molecular physics leads to a similar development.

The four main motivations in cold molecules research can be summarized as i) high-resolution spectroscopy and precision spectroscopy, ii) the study of longrange interactions, in particular between polar molecules, iii) quantum information processing, and iv) chemical reaction dynamics. ${ }^{[2]}$ High-resolution spectroscopy

${ }^{*}$ Correspondence: Prof. Dr. A. Osterwalder Institut des Sciences et Ingénierie Chimiques EPFL-SB

$\mathrm{CH}-1015$-Lausanne

Tel.: +41216937971

Fax: +4121693 7970

E-mail: andreas.osterwalder@epfl.ch (point i) above) benefits in two ways from low temperatures: First, a reduction of the internal temperature condenses the state population in the lowest levels, thus reducing spectral congestion. ${ }^{[3]}$ Second, low velocities in the laboratory frame-ofreference (FR) and the increased observation times that follow, directly increase the maximum possible resolution. ${ }^{[4]}$ Longrange interactions (point ii)) have their origin in the interaction between charged or polar (dipole and higher multipoles) particles, and in the atomic and molecular polarizabilities. They are mostly attractive but significantly weaker than the short-range forces that govern most of the room-temperature chemistry, and they are thus best studied in low-energy collisions. Similarly, if the molecular motion can be well-controlled, and the molecules can be confined in a well-defined environment, controlled interactions can be used for information storage and processing (point iii)). ${ }^{[5]}$ Chemical reaction dynamics (point iv)) benefits in different ways from cold molecules: The narrow velocity distribution in a packet of cold molecules provides high resolution in collision studies, also at high collision energies. The detailed study of chemical reactions at temperatures of a few Kelvin is of great interest in particular also for an understanding of interstellar chemistry. Completely new aspects become accessible, however, if the velocities in the molecular FR are reduced. Fundamental effects like tunneling or scattering resonances in chemical reactions can be best studied with only few angular momentum partial waves populated, and pure s-wave scattering can only be observed at the very lowest temperatures. Finally, the failure of all classical models is expected when the DeBroglie wave length of the reactants becomes comparable to the intermolecular distances during reaction. First collisions between decelerated molecules and atoms were observed in 2006, ${ }^{[6]}$ and despite great efforts, only very few additional studies have been published since, mainly due to the limited particle density. ${ }^{[7,8]}$

All these areas require methods to control molecular motion and temperature, and during the past 15 or so years several techniques have been developed to achieve this goal (see the individual chapters in ref. [2] for details). Broadly speaking the available methods can be divided into a first category where the molecules are assembled from ultracold atoms, and a second one where a molecule of interest is controlled directly. In the first one, ultracold atoms, cooled by standard atom cooling methods, are assembled to molecules using electric, magnetic, or optical fields. These methods currently provide the lowest temperatures but so far are restricted to alkali metals. The second category includes a variety of methods ranging from collisions with a cold buffer gas to a backward-rotating nozzle or collisions of the target molecule in crossed beams. A particularly powerful method has emerged from the exploitation of the Stark effect, the energy change of polar molecules in electric fields. The resulting method, first demonstrated in 1999, is generally called Stark deceleration, ${ }^{[9,10]}$ and is at the basis of the work presented here. Equivalent approaches are also available for paramagnetic atoms or molecules, using magnetic fields, and this leads to socalled Zeeman-deceleration. ${ }^{[11]}$

The Stark effect is the shifting and splitting of energy levels in an electric field. As an example, Fig. 1A shows the Stark effect in the $\mathrm{J}_{\mathrm{K}}=5_{5}$ level of the $\mathrm{ND}_{3}$ molecule. At zero field, the level is split in the two components of the inversion doublet, and as the field increases both levels split and fan out each into $\mathrm{J}+1$ levels that are labelled by the quantum number $\mathrm{M}$, the 

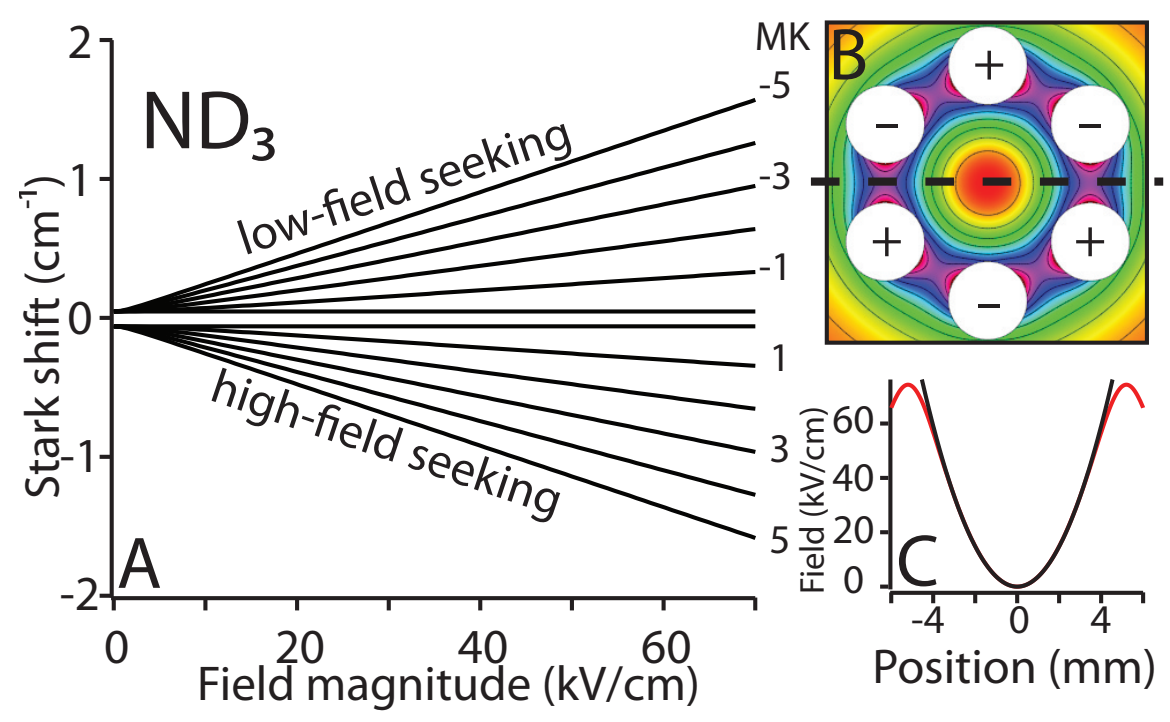

Fig. 1. A) Stark effect of the $\mathrm{J}=5, \mathrm{~K}=5$ rotational level of $\mathrm{ND}_{3}$. B) Electric field distribution obtained when arranging 6 electrodes on a hexagon and applying alternating positive and negative voltage. C) Red line: cross section through the distribution in panel B, for the case of $\pm 8 \mathrm{kV}$. The black line shows a parabola fitted to the actual potential.

projection of the total angular momentum vector on the electric field axis. Since the level-energy changes with field, a spatially inhomogeneous electric field produces a force proportional to the slope of the line in Fig. 1A. Levels with positive slope (negative slope) feel a force towards regions with lower (higher) electric fields and are thus called low-field-seeking (high-fieldseeking) states. Particular arrangements of high-voltage electrodes enable the production of very strong electric fields $(>100$ $\mathrm{kV} / \mathrm{cm}$ ) that also provide the field gradients necessary to control the velocity of the polar molecules.

For example, one can arrange an even number of electrodes on a circle and apply alternating positive and negative high voltage to them, as is shown in Fig. 1B. This produces an electric field minimum at the center of the structure, as shown in Fig. 1C, the cross section through the potential from Fig. 1B for the case of $\pm 8 \mathrm{kV}$ on the electrodes. Low-field-seeking (lfs) states will be attracted to the central region of this structure, high-field-seeking states (hfs) will be repelled by it. Such a structure can be extended perpendicular to the plane of the figure in order to obtain a guide for neutral molecules that move along its axis (see section 3 ). The forward velocity of the molecules is controlled if a field gradient is produced perpendicular to the propagation direction of the molecules. An electric field minimum can also be produced in three dimensions, thus providing a trap where polar neutral molecules can be confined. Molecular samples with temperatures of $\approx 500 \mathrm{mK}$ can thus be stored for up to several seconds, currently limited by collisions with background molecules, and by transitions to un-trapped states by blackbody radiation. ${ }^{[12]}$ In the following sections, efforts at EPFL are described to develop and improve methods to reach the ultimate control over both internal and external degrees of freedom in neutral molecules, and plans for applications. Section 2 describes a decelerator where the molecules are picked up and carried through a structure in moving three dimensional (3D) electrostatic traps. Section 3 describes how an electrostatic guide can be used to filter the slow molecules from a thermal sample, and how the sample emerging from such a filter is composed in terms of velocities and rotational state populations. Both these methods are developed and characterized in view in particular of applications to high-resolution low-temperature collision studies between neutral molecules.

\section{Deceleration of Polar Neutrals in Traveling Traps}

In this section a method is described that was recently developed for the deceleration of polar neutral molecules in moving 3D electrostatic traps. ${ }^{[13]}$ As described in the previous chapter, an inhomogeneous electric field of the type shown in Fig. 1B can be used to guide polar molecules along the axis of an electrode array. A series of ring-electrodes can be used to generate a similar field, but with a 3D minimum, constituting a trap for polar molecules. Molecules can be confined inside the trap if their velocity relative to the trap center is sufficiently low. Similar concepts have been used previously for the construction of a microstructured Stark decelerator, and for the deceleration of Rydberg atoms and molecules. ${ }^{[14,15]}$

The basis of the setup is the arrangement of ring-electrodes along the axis of a cylinder, as shown in cross section in Fig. $2 \mathrm{~A}$. The decelerator for polar neutral molecules is generated as follows: A sinusoidal potential along the structure produces two 3D electrostatic traps per period. Fig. 2A sketches the electrode structure and the sine potential (red line); the resulting electric field distribution is shown in Fig. 2C where the cylinder axis is the $\mathrm{X}$-axis of each panel. In Fig. 2C, the longitudinal change of the electric field magnitude on the cylinder axis is shown above each of the contour plots. Contour lines start at, and are spaced by $4 \mathrm{kV} / \mathrm{cm}$, and the dashed (bold) lines show $28 \mathrm{kV} / \mathrm{cm}(16 \mathrm{kV} / \mathrm{cm})$ for reference. The voltage on each electrode is given by $\mathrm{V}_{\mathrm{n}}=\mathrm{V}_{0} \sin \left(\phi_{0}+2 \pi \mathrm{n} / 8\right)$, where $\mathrm{V}_{0}$ is the sine amplitude, and $\mathrm{n}$ is the number of the electrode. The shift $2 \pi \mathrm{n} / 8$ per electrode is due to the periodicity of 8 in the current setup. $\phi$ is an overall phase offset which is 0 in plot II of panel $2 \mathrm{C}$ and $\pi / 8$ in plot I. This difference in $\phi_{0}$ leads to a shift of the traps from in-plane with an electrode to between two electrodes. These two configurations demonstrate that a time-dependence of $\phi_{0}$ leads to a displacement of the traps, and indeed, a continuous increase of $\phi_{0}$ leads to a continuous motion of the traps through the structure. In order to obtain this dependence of $\phi_{0}$, the voltage on each of the electrodes must be modulated sinusoidally in time, with a phase-shift of $\pi / 4$ between adjacent electrodes, as is shown by the green sine-plots in Fig. 2A. The modulation frequency $\omega$ determines the speed of the traps: Increasing $\phi_{0}$ by $2 \pi$ shifts the traps by the length of one period, $L$, and the velocity is thus given by $\mathrm{v}_{\text {trap }}=\omega / 2 \pi L$.

Recently, the first decelerator of this type has been demonstrated by decelerating CO molecules. ${ }^{[13]}$ Fig. 2B shows a sketch of the setup used there: A cooled, pulsed, supersonic expansion (bottom of the panel) produces packets of $\mathrm{CO}$ with a center velocity around $300 \mathrm{~m} / \mathrm{s}$ at a repetition rate of $10 \mathrm{~Hz}$. The expansion is crossed by a laser to produce the metastable $\mathrm{a}^{3} \Pi_{1}(\mathrm{v}=$ $0, \mathrm{~J}=1, \mathrm{M} \Omega=-1$ ) state in CO. These molecules enter the $480 \mathrm{~mm}$ long decelerator where they are captured by the traps and carried through the structure. At the end of the decelerator the molecules are detected on a multichannel plate assembly, and their flight time is measured. Sine-waveforms with up to $20 \mathrm{kV}$ peak-to-peak amplitude can be applied to the electrodes with frequencies currently tunable between $\approx 12-$ $30 \mathrm{kHz}$, corresponding to an accessible range of velocities between $144 \mathrm{~m} / \mathrm{s}$ and $360 \mathrm{~m} / \mathrm{s}$, given by the current period length of $12 \mathrm{~mm}$. 


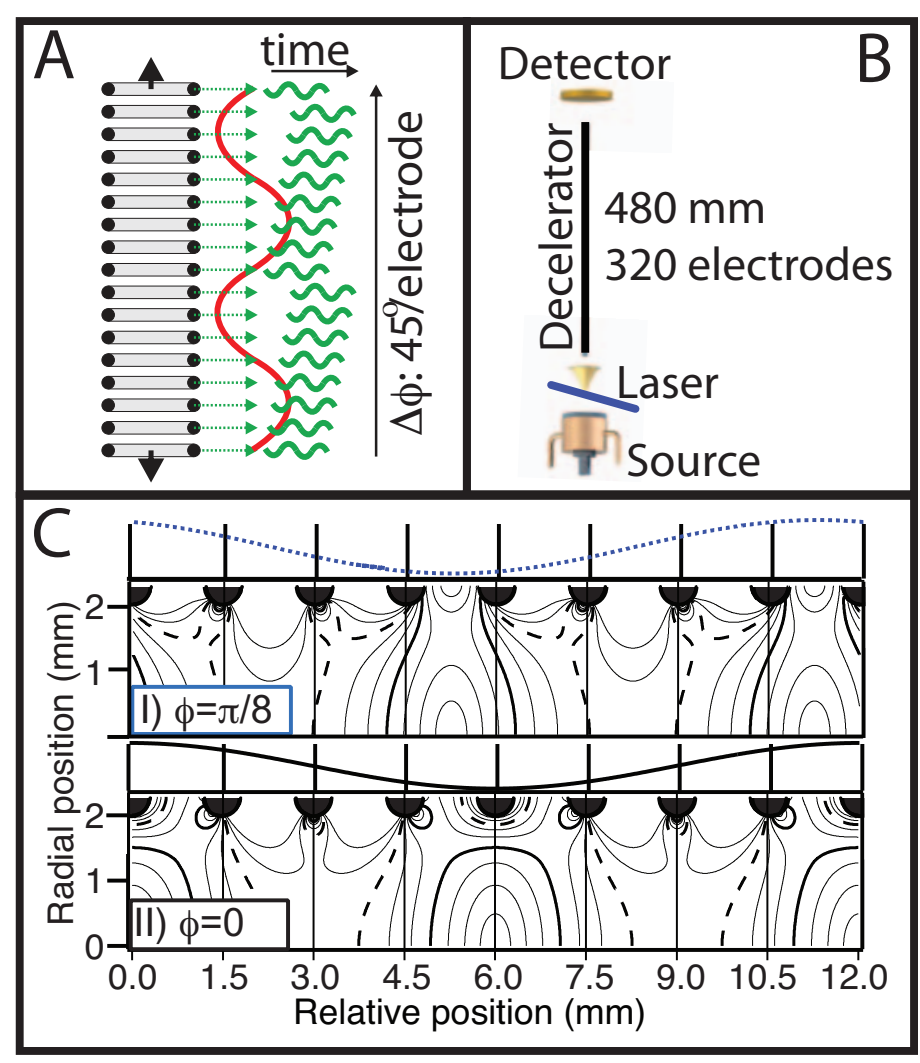

Fig. 2. A) Section of the electrode array used for the 3D trap decelerator. The red curve is the spatial sine required to produce the traps, the green lines show the temporal variation of the voltages on individual electrodes that move the traps through the decelerator. B) Sketch of the setup. C) Contour plots of the electric field magnitude inside the electrode structure for offsets $\phi_{0}=0$ (panel II) and $\phi_{0}=\pi / 8$ (panel I). In both panels, the cylinder axis is at the bottom of the plot, and the curves above the plots show the electric field magnitude on axis.

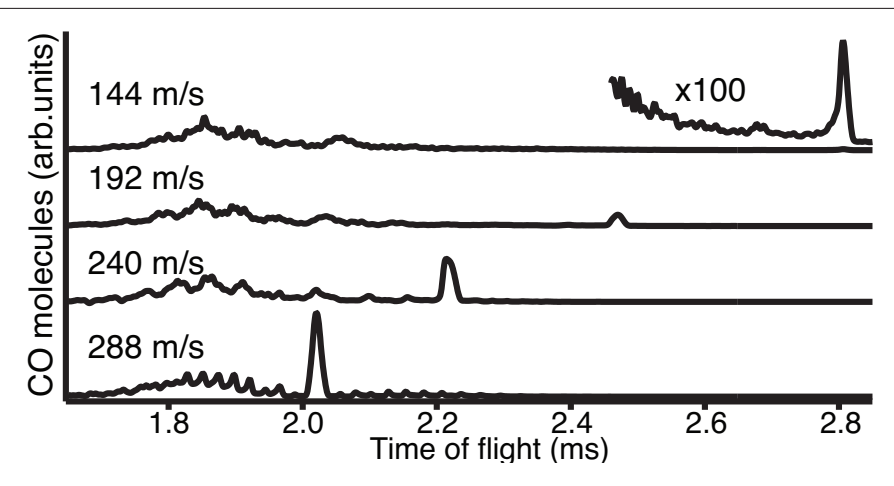

Fig. 3. Deceleration of $\mathrm{CO}$ molecules. The molecules are picked up at $288 \mathrm{~m} / \mathrm{s}$, and guided through the structure at constant velocity (bottom trace), or decelerated to $240 \mathrm{~m} / \mathrm{s}, 192 \mathrm{~m} / \mathrm{s}$ or $144 \mathrm{~m} / \mathrm{s}$ (second to fourth trace, from below).

Time-of-flight traces are shown in Fig. 3. In all these traces, the traps were initially moving at $288 \mathrm{~m} / \mathrm{s}$. With a constant frequency those molecules with velocities near $288 \mathrm{~m} / \mathrm{s}$ are actively guided through the structure with high efficiency, while those with different velocities are not confined. Reduction of the modulation frequency slows down the traps and thus also the molecules. The three upper traces in Fig. 3 demonstrate this by decelerating CO to 240,192 , and $144 \mathrm{~m} / \mathrm{s}$, respectively. These first measurements show a clear signal even when $75 \%$ of the kinetic energy are removed.

Three of the main advantages of this approach over traditional Stark decelerators are that i) it captures a significantly larger volume of molecules in six-dimensional phase-space (defined by the three velocity and three spatial coordinates), even when compared to the best currently operational Stark decelerator;[16] ii) the molecules are always trapped in true 3D traps, which leads to higher efficiency of the transportation process through the guide; iii) the molecules do not need to be explicitly loaded into traps; and iv) the field gradients are very strong even at low electric fields. The first three points are important since in most experiments higher number density is important, and many of the areas of interest described in section 1, including in particular also collision studies, are currently limited by the available particle density. The fourth point is of interest for the particular application to heavy diatomic molecules like $\mathrm{SrF}$ or YbF. Such molecules are currently being used in precision-spectroscopy studies to measure the permanent electric dipole moment of the electron. ${ }^{[17]}$ The individual rotational levels of such molecules have lfs components at low electric fields, but due to the low rotational constants even at moderate field strengths all rotational levels undergo avoided crossings and become hfs, and they can not be decelerated in a conventional Stark decelerator. Using the method presented here, these molecules can be decelerated since they never enter regions with strong fields, and they thus always stay lfs.

\section{Filtering of Polar Neutrals in Electrostatic Guides}

An alternative approach for the preparation of cold molecules has been developed in 2003 by Rangwala et al., and it is based on the realization that slow molecules are present also in a thermal sample. ${ }^{[18]}$ A cold sample can be obtained by filtering them out of a higher temperature gas. The implementation of such a filter can be done for example by using an electrode arrangement as depicted in Fig. 1B to build a curved guide. In the bend, the molecules feel both the confining force from the guide and the centrifugal force due to the curvature. The centrifugal force depends on the forward velocity, and above a certain threshold it is stronger than the Stark force. Molecules with forward velocities above this threshold are lost from the guide. The threshold can be adjusted by setting the voltage on the electrodes; by choosing the upper threshold velocity, the temperature of the sample at the end of the guide is controlled.

Since the first demonstration, filters of this type have been used in collision studies for ion-molecule reactions, ${ }^{[19]}$ and they have been demonstrated to work with a variety of molecules. The interest here is to use velocity filters for neutral-neutral collision studies, by combining a velocity filter with another source in crossed or merged beams. It is crucial in such studies to know the rotational state populations, and it is thus important to understand how the filtering affects different rotational states. Initially, the temperature of the molecules is the same as that of the source. In most current velocity-filter experiments, the filtered molecules are injected into the guide from an effusive source that is either operated at room-temperature or slightly cooled. Cryogenic sources have been used in combination with velocity filters, but they were shown to be more difficult to operate than a normal effusive source. ${ }^{[20]}$

The effective dipole moment, which is the change in energy with electric field, depends both on the permanent electric dipole moment of a molecule and its rotational quantum numbers. Since the threshold velocity for guiding depends on the Stark force, it depends on the effective dipole moment and is thus different for each rotational level. As a consequence, the rotational state distribution of the filtered molecules will be different from the initial distribution. Previously, depletion mea- 
surements have been performed to obtain information on the relative contributions of different states. ${ }^{[21]}$ Recently, state-selective resonance-enhanced multi-photon ionization (REMPI) has been used to obtain the complete rotational spectrum of the guided molecules as well as velocity distributions for individual rotational levels. ${ }^{[22]}$

Fig. 4 shows the result from a typical velocity-filter experiment to assess the velocity distribution of the filtered molecules: here, the molecule density is plotted as a function of the delay time between switching on of the guide and firing of the pulsed laser for detection. The source runs continuously, and the measured density gives information about the probability density for a particular velocity: at short delay times, only the highest velocities contribute, and ever lower velocities appear at longer delays. From the shape of the curves in Fig. 4 one can obtain the velocity distribution via a derivative, and the result is a curve similar to those shown in the inset. The solid, dashed, and dash-dotted lines in the main panel show the density distribution for the rotational levels $J_{K}=9_{9}, 4_{3}$, and 3 . These distributions are well reproduced by trajectory simulations, as shown by the dotted line under each trace. The velocity distributions obtained from the simulations are shown in the inset for the different levels, using the same line types. Each level, due to the different effective dipole moment, is represented in the final distribution at different velocities, even though they all start with the same, $300 \mathrm{~K}$, distribution.

In an alternative experiment, the detection time is fixed and the laser wavelength is scanned to record an entire vibrational band in the guided molecules. Via the different intensities in such a spectrum, (one is shown in Fig. 5) the different guiding probabilities for the different rotational levels are obtained. The upper panel in Fig. 5 shows an overview of the $\mathrm{B}\left(\mathrm{v}_{2}=5\right)$ $\leftarrow \mathrm{X}(1)$ band of $\mathrm{ND}_{3}\left(\mathrm{v}_{2}\right.$ is the umbrella motion in ammonia, $\mathrm{X}(1)$ designates the upper component of the inversion doublet in the ground state) and the lower panel shows a small section in the center of that band. The solid line is the spectrum obtained for ammonia molecules from the background gas, with the guide switched off, and they thus show the normal roomtemperature spectrum. The dashed line shows the spectrum for the guided molecules. Open (closed) circles in the stickspectrum show the calculated intensities for the transitions of background (guided) molecules. The effective dipole moment is the highest for levels with $\mathrm{J} / \mathrm{K}$ close to 1 , for example the $6_{6}$ or the $9_{9}$ levels in Fig. 5. Those levels with very large rations $\mathrm{J} / \mathrm{K}$ have small effective dipole moments and are not guided efficiently. The traces shown in Fig. 4 are recorded

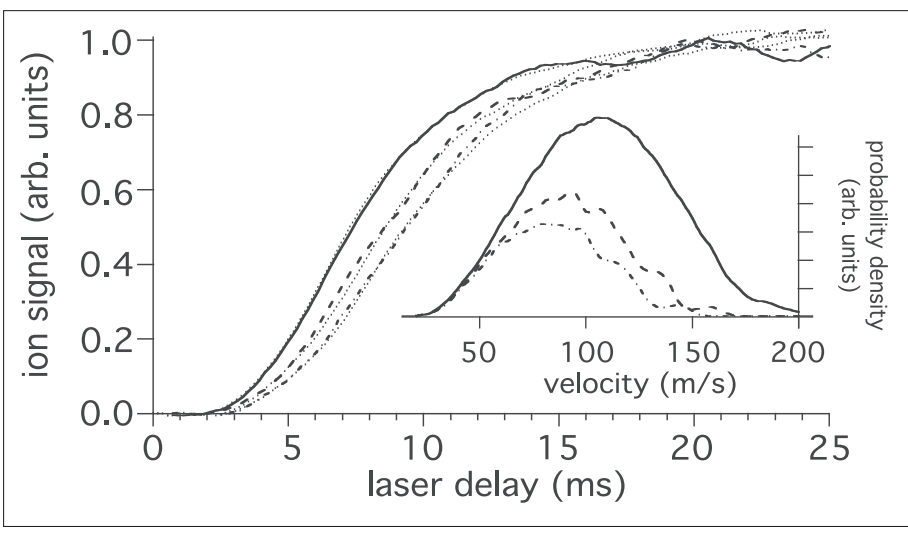

Fig. 4. Ammonia molecule density at the end of the velocity filter as a function of delay time between switching on the guide and firing the detection laser. The solid, dashed, and dash-dotted lines show the results for the rotational levels $J_{K}=9_{9}, 4_{3}$, and $3_{2}$. The dotted lines are results of trajectory simulations. The inset shows the velocity distributions for the three rotational states, using the same line types, as extracted from the simulations.

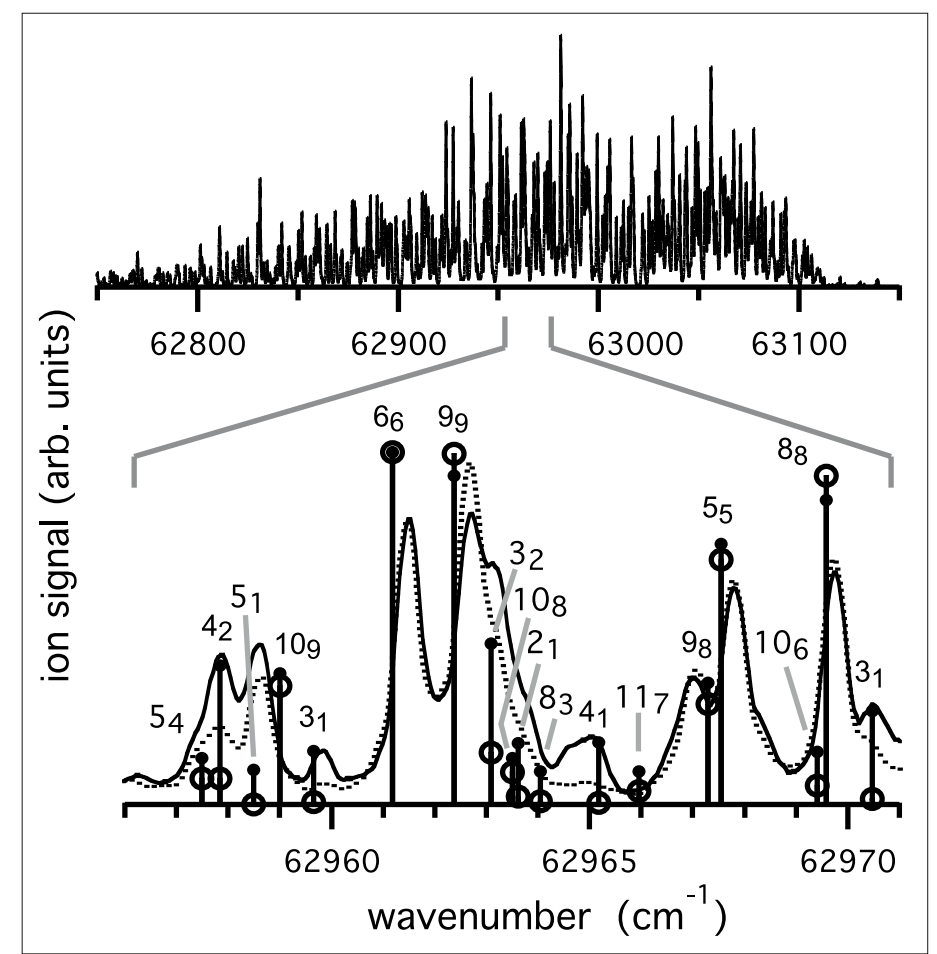

Fig. 5. Top panel: Overview REMPI-spectrum of the $B\left(v_{2}=5\right) \leftarrow X(1)$ band of $\mathrm{ND}_{3}$. Bottom panel: small section from the overview spectrum. The solid line shows the result for background molecules with the guides switched off, the dotted lines for the guided molecules. Open and closed circles are the calculated intensities for the respective transitions without and with guide, respectively. Labels in the spectrum designate the rotational state $J_{K}$ for the initial state of the respective transition.

by parking the detection laser on one of the transitions shown in Fig. 5 and scanning the detection time. From Figs 4 and 5 it becomes evident that not only does the rotational temperature change in the sample - even if it does not change the same way as the translational temperature; also the translational temperature in the guided sample is different for each rotational level. Quantitative information on these distributions is a crucial prerequisite for the understanding of collision studies involving velocity-filtered molecules.

It has recently been demonstrated[22,23] how the properties of the filter can be further improved by implementing a velocity band-pass filter, as opposed to the low-pass filter obtained by the normal arrangement: by using a guide that is segmented into independent components, an electrostatic beam-chopper can be implemented which can be used to discriminate also against 


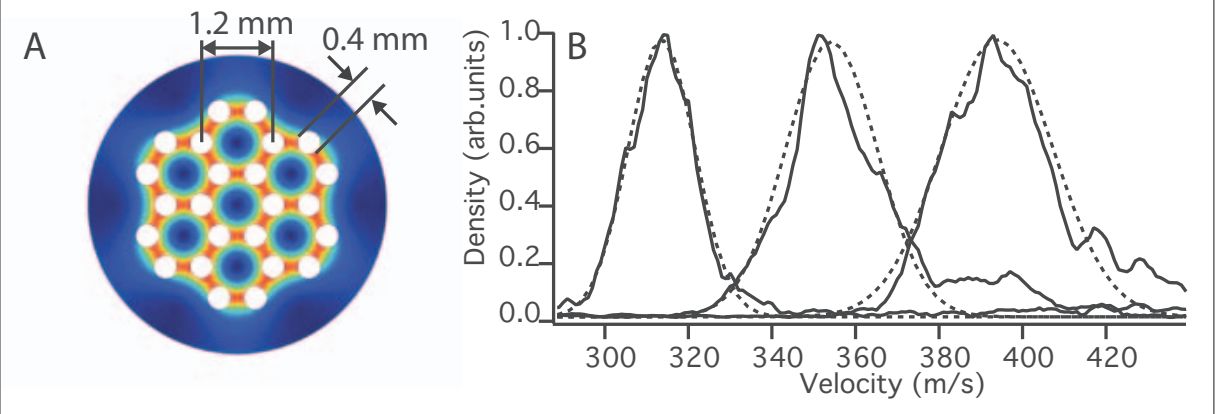

Fig. 6. Panel A: Cross section through the honeycomb array of miniaturized electrostatic guides. White circles are electrodes, red (blue) is strong (weak) electric field. Panel B: Simulated velocity distributions obtained using a segmented guide based on the field from panel A. Dashed lines are Gaussians to guide the eye.

slow molecules. Currently, work on an extension of this concept in combination with a guide that enables the filtering of faster molecules as well is in progress: the upper velocity-limit for the guided molecules is defined by the field gradient that can be produced in the curved section, and in the guide used here that limit is around $250 \mathrm{~m} / \mathrm{s}$ for $\mathrm{ND}_{3}$. Using a similar geometry but with all dimensions scaled down by a factor of 10 one can obtain a guide with a similar depth of the potential, but with significantly stronger field gradients. The concept to achieve this is sketched in Fig. 6: the scaled-down guide produces electric fields between the different electrodes that are similar to the ones in the present guide, but because the inner diameter of the guide is smaller, the electric field gradient is stronger. Scaling down all dimensions reduces the active surface of the guide, and to obtain the same overall flux one has to use many guides in parallel, for example by arranging them in a honeycomb structure as shown in Fig. 6A. The resulting structure enables the filtering of molecules significantly faster than $300 \mathrm{~m} / \mathrm{s}$ and therefore allows the use of a supersonic expansion for the production of the molecular beam. These molecules will thus be internally cooled in the expansion, and a segmented, miniaturized guide will then be used to filter out a narrow band from the supersonic expansion as shown in Fig. 6B. The width of these distributions corresponds to translational temperatures below IK. This type of filter will enable merged-beam experiments, on neutral molecules, by combining it for example with other filters, or with direct expansions.

\section{Acknowledgments}

The project described in section 2 is done in collaboration with Prof. Dr. Gerard Meijer (department of molecular physics at the FritzHaber-Institute of the Max-Planck-Society, Berlin). Financial support from the EPFL and the Swiss National Science Foundation through grant number PP0022_119081 are greatly acknowledged.

Received: June 6, 2011

[1] S. Y. T. van de Meerakker, H. L. Bethlem, G. Meijer, Nat. Phys. 2008, 4, 595.

[2] R. Krems, B. Friedrich, W. C. Stwalley, 'Cold Molecules: Theory, Experiment, Applications',
CRC Press, Boca Raton, FL, 2009.

[3] T. Rizzo, J. Stearns, O. Boyarkin, Int. Rev. Phys. Chem. 2009, 28, 481

[4] J. van Veldhoven, R. T. Jongma, B. G. Sartakov, W. A. Bongers, G. Meijer, Phys. Rev. A 2002, 66, 032501.

[5] A. André, D. DeMille, J. M. Doyle, M. D. Lukin, S. E. Maxwell, P. Rabl, R. J. Schoelkopf, P. Zoller, Nat. Phys. 2006, 2, 636.

[6] J. J. Gilijamse, S. Hoekstra, S. Y. T. van de Meerakker, G. C. Groenenboom, G. Meijer, Science 2006, 313, 1617.

[7] L. Scharfenberg, J. Kłos, P. J. Dagdigian, M. H Alexander, G. Meijer, S. Y. T. van de Meerakker, Phys. Chem. Chem. Phys. 2010, 12, 10660.

[8] B. Sawyer, B. Stuhl, D. Wang, M. Yeo, J. Ye, Phys. Rev. Lett. 2008, 101, 203203.

[9] H. L. Bethlem, G. Berden, G. Meijer, Phys. Rev. Lett. 1999, 83, 1558 .

[10] H. L. Bethlem, G. Meijer, Int. Rev. Phys. Chem. 2003, 22, 73

[11] N. Vanhaecke, U. Meier, M. Andrist, B H. Meier, F. Merkt, Phys. Rev. A 2007, 75 031402(R).

[12] S. Hoekstra, J. Gilijamse, B. Sartakov, N. Vanhaecke, L. Scharfenberg, S. Van de Meerakker, G. Mejer, Phys. Rev. Lett. 2007, 98, 133001.

[13] A. Osterwalder, S. A. Meek, G. Hammer, H. Haak, G. Meijer, Phys. Rev. A 2010, 81, 051401.

[14] S. A. Meek, H. Conrad, and G. Meijer, Science 2009, 324, 1699.

[15] S. D. Hogan, C. Seiler, F. Merkt, Phys. Rev Lett. 2009, 103, 123001.

[16] L. Scharfenberg, H. Haak, G. Meijer, S. Y. T. van de Meerakker, Phys. Rev. A 2009, 79, 023410.

[17] M. R. Tarbutt, J. J. Hudson, B. E. Sauer, E. A. Hinds, Faraday Discuss. 2009, 142, 37.

[18] S. A. Rangwala, T. Junglen, T. Rieger, P. W H. Pinkse, G. Rempe, Phys. Rev. A 2003, 67, 043406

[19] M. T. Bell, A. D. Gingell, J. M. Oldham, T. P. Softley, S. Willitsch, Faraday Discuss. 2009, 142, 73.

[20] L. D. Van Buuren, C. Sommer, M. Motsch, S. Pohle, M. Schenk, J. Bayerl, P. W. H. Pinkse, G. Rempe, Phys. Rev. Lett. 2009, 102, 033001.

[21] C. Sommer, L. D. Van Buuren, M. Motsch, S. Pohle, J. Bayerl, P. W. H. Pinkse, G. Rempe, Faraday Discuss. 2009, 142, 203.

[22] B. Bertsche, A. Osterwalder, Phys. Rev. A 2010 82, 033418.

[23] C. Sommer, M. Motsch, S. Chervenkov, L. D. V. Buuren, M. Zeppenfeld, P. W. H. Pinkse, G. Rempe, Phys. Rev. A 2010, 82, 013410. 